\title{
On the Stability of the Differentiability of Cauchy Horizons
}

\author{
Piotr T. Chruściel AND JAmes Isenberg
}

\begin{abstract}
We prove that for spacetimes containing compact Cauchy horizons which satisfy certain conditions, the existence and differentiability of the horizon is stable under smooth perturbations of the metric. The arguments of the proof suggest, however, that if these conditions are not imposed then the Cauchy horizon and its differentiability are not necessarily stable.
\end{abstract}

\section{Introduction.}

An important global feature of spacetimes is the existence - or absence - of horizons of various kinds, such as Cauchy horizons, event horizons, and observer horizons. Their occurrence signals various dramatic properties: For example, a spacetime with a Cauchy horizon is generally nondeterministic (see, e.g., [Ch] for a review), while an event horizon implies the existence of a black hole. A good understanding of the properties of horizons turns out, therefore, to be one of the fundamental issues for understanding the global properties of spacetimes.

Most results concerning horizons - e.g., the black hole area theorem [Haw1], the topology of black holes theorem [Haw2], Hawking's chronology protection results [Haw3], the isometry results for solutions with compact Cauchy horizons [M-I], [I-M] - are proved assuming a rather high degree of differentiability; typically $C^{2}$ or higher is needed. It is therefore natural to ask whether such a degree of differentiability of horizons should be expected in a generic spacetime.

Recall that, because of their achronal character, every horizon must locally be the graph of some Lipshitz continuous function $[\mathrm{P}]$. It follows from Rademacher's Theorem [Fe] that horizons are differentiable almost everywhere. The tangent planes are, however, not guaranteed to vary continuously from point to point; this result does not even guarantee that a horizon 
is almost everywhere $C^{1}{ }^{1}$ Yet as mentioned above, for results like the area theorem, at least $C^{2}$ differentiability is needed. We should stress that this issue is rather distinct from that of the differentiability of the spacetime metric; for the purposes of this discussion, we shall assume that the metric is $C^{\infty}$.

In spite of the fundamental character of the issue of the differentiability of Cauchy horizons, very little seems to be known about it. It has been shown [Ch-I1] that asymptotically flat, compactly generated ${ }^{2}$ Cauchy horizons cannot be $C^{1}$. Nondegenerate Killing horizons are easily shown to be smooth (or, at least, of the same degree of differentiability as the metric), but nothing is known about the differentiability of degenerate Killing horizons. It has been argued that Cauchy horizons with fountains ${ }^{3}$ are smooth in a neighborhood of the fountains; however this is not yet clear, and nothing is known about the differentiability of such a horizon away from the fountains.

As a first step towards understanding this problem, we wish to consider the following:

Question. Let $m$ be a fixed positive integer. Let $\hat{\mathcal{H}}$ be a $C^{k}$ (for $\left.1 \leq k \leq \infty\right)$ horizon in a spacetime $(M, \hat{g})$ with a $C^{k-1}$ metric. Is it true, for all (or some, or most) $C^{k-1}$ metrics $g$ in a neighborhood of the metric $\hat{g}$, that the corresponding horizon $\mathcal{H}=\mathcal{H}(g)$ - if it exists - will be of differentiability class $C^{m}$ ?

We shall show that, at least for a certain (rather restricted) class of spacetimes, the answer to this question is yes. The spacetimes $(M, \hat{g})$ in this class are characterized by the requirement that each contains a compact Cauchy horizon whose null generators admit a global Poincaré section. Then if a certain global quantity which we call $Q$ is sufficiently large, and if the spacetime differentiability order $k-1$ is sufficiently large as well, stability follows.

This result provides a partial answer to our question. However, it is important to recognize its limitation. In particular, we note that while our argument depends on the hypothesis that the Cauchy horizon be compact

\footnotetext{
${ }^{1}$ Recall that a function is $C^{1}$ if it is differentiable at each point and if its derivative is a continuous function.

${ }^{2} \mathrm{~A}$ Cauchy horizon is compactly generated if all its generators, when followed into their past, enter and remain in a compact subset. See [Haw3].

${ }^{3} \mathrm{~A}$ Cauchy horizon contains a fountain if at least one of its generators is closed, and if almost all of its generators, when followed into their past, asymptotically approach a closed generator. See [Th].
} 
and that the generators of this horizon admit a global Poincaré section, we have no reason to believe that these hypotheses are needed for the stability of the horizon. Note, however, that if we consider a spacetime whose Cauchy horizon does have a Poincaré section then we believe that large $Q$ is needed for stability. These limitations need much further study.

It should be pointed out that the question we have raised here, as well as the partial answer which our result provides, do not involve any field equations. Hence, as we will show, it is easy to construct open sets of spacetimes which do satisfy our stability criterion, as well as open sets of spacetimes which do not. So our study is really one which concerns Lorentzian geometry, rather than one which concerns general relativity and Einstein's equations. It is also relevant to the discussion of what an "arbitrarily advanced civilization" (see [Th]), with some control of energy conditions in its spacetime, can do.

If we focus on spacetimes which satisfy the Einstein (or similar) field equations, an appropriate question to consider is that of the stability of horizons with small perturbations of the Cauchy data on a partial Cauchy surface. However, if one takes the strong cosmic censorship conjecture seriously, then one expects that most Cauchy data perturbations will lead to large changes of the spacetime metric in a neighborhood of the Cauchy horizon (with the horizon unlikely to survive), so that our question seems to be irrelevant from this point of view. Note, however, that the strong cosmic censorship conjecture is still an open issue. Moreover, it is of some interest to understand what happens to the Cauchy horizon under those, presumably nongeneric, perturbations of Cauchy data for which the spacetime metric perturbations are actually small in a neighborhood of the horizon. (Such perturbations are well-known to exist; see, e.g., [M], [C-I-M].) As our results are valid for all small perturbations of the spacetime metric, in particular they apply as well to those which satisfy certain field equations. Thus our results do have some relevance to general relativity, at least for spacetimes which contain a compact Cauchy horizon satisfying our structure and stability conditions.

\section{The Instability of Some Cauchy Horizons.}

Before stating and proving our main result concerning the stability of differentiability for Cauchy horizons in certain spacetimes, we wish to show here that without at least some restrictions on the spacetime, the existence of a Cauchy horizon is not a stable property under metric perturbations. We 
do this via the following example. (See also [Be] for a similar discussion.)

Let ${ }^{2} M$ be any compact two-dimensional manifold and set ${ }^{4} M=\mathbb{R} \times$ $S^{1} \times{ }^{2} M$. Let $g$ be the metric on ${ }^{4} M$ which is given in local coordinates for $t \in \mathbb{R}, \phi \in S^{1}$, and $x^{a} \in{ }^{2} M$ - by

$$
g=2 d t d \phi+t^{2} d \phi^{2}+h_{a b} d x^{a} d x^{b},
$$

where $h=h_{a b} d x^{a} d x^{b}$ is a Riemannian metric on ${ }^{2} M$, independent of $\phi$ and $t$. It is easily seen that the two spacetime regions $\left({ }^{4} M_{ \pm}, g \mid{ }_{4} M_{ \pm}\right)$in $\left({ }^{4} M, g\right)$, where

$$
{ }^{4} M_{ \pm}:=\left\{p \in{ }^{4} M \mid \pm t(p)>0\right\},
$$

are globally hyperbolic. One also verifies that the hypersurface

$$
\mathcal{H}:=\left\{p \in{ }^{4} M \mid t(p)=0\right\}
$$

is a Cauchy horizon.

Now consider the family of $\varepsilon$-parametrized (for $\varepsilon>0$ ) spacetimes $\left({ }^{4} M, g_{\varepsilon}\right)$, with ${ }^{4} M$ as above, and with the metric $g_{\varepsilon}$ given by

$$
g_{\varepsilon}=2 d t d \phi+\left(t^{2}+\varepsilon\right) d \phi^{2}+h_{a b} d x^{a} d x^{b} .
$$

These spacetimes are all globally hyperbolic, with no Cauchy horizons. (This is easily verified by noting that $g_{\varepsilon}^{-1}(d t, d t)=-\left(t^{2}+\varepsilon\right)$, so that $t$ is a timefunction on ${ }^{4} M$. Global hyperbolicity then immediately follows from compactness of the level sets of $t$.) So, taking $\varepsilon$ arbitrarily small, we see that there are arbitrarily small perturbations of $\left({ }^{4} M, g\right)$ which result in globally hyperbolic spacetimes, with no Cauchy horizon. Note, too, that if we multiply $\varepsilon d \phi^{2}$ by a compactly-supported non-negative function $\psi$, with $\left.\psi\right|_{\mathcal{H}}>0$, then we have compactly supported metric perturbations of $(M, g)$ which also remove the Cauchy horizon.

So unless one makes further restrictions, the presence of a Cauchy horizon in a spacetime need not be a stable property under metric perturbations. We shall make such restrictions in formulating our main result, in the next section.

\section{Main Result on Stability.}

Let us consider a $C^{\ell}(\ell \geq 1)$ spacetime $\left({ }^{4} M, g\right)$ which contains a connected, compact (say, future) Cauchy horizon $\mathcal{H}$ which is a $C^{k}(k \geq 1)$ differentiable submanifold of ${ }^{4} M$. One of the conditions we will impose involves 
the existence of a Poincaré section. We say that $\mathcal{H}$ has a global Poincaré section (or, for short, a "section") if it contains a surface ${ }^{2} M$ such that a) every null generator of $\mathcal{H}$ intersects ${ }^{2} M$ at least once, and b) the generators always intersect ${ }^{2} M$ transversally. One readily verifies that if $\mathcal{H}$ is compact, and if $\mathcal{H}$ has a section as just described, then there exists a diffeomorphism $\Psi$ of ${ }^{2} M$ such that

$$
\mathcal{H} \approx S^{1} \times_{\Psi}{ }^{2} M=\left([0,1] \times{ }^{2} M\right) / \sim,
$$

where the equivalence " $\sim$ " is defined by

$$
(1, p) \sim(0, \Psi(p)) .
$$

$\Psi$ is called the Poincaré map of the null generators of $\mathcal{H}$.

The other essential condition we will need to impose for our main result involves a quantity we call $Q$. To define $Q$, [see equation (11)], we need some preliminaries:

Given a Cauchy horizon $\mathcal{H}$ with section ${ }^{2} M$, for $p \in{ }^{2} M$ we define $\Gamma(p)$ to be the path of the null generator which starts at $p$ and ends at the next intersection this generator makes with ${ }^{2} M$, at the point $\Psi(p)$. So $\Gamma(p) \cap{ }^{2} M=\{p, \Psi(p)\}$.

Now let $\varphi$ be any defining function for $\mathcal{H}$ in ${ }^{4} M$ (that is, $\varphi(p)=0$ if and only if $p \in \mathcal{H}$, and $d \varphi(p) \neq 0$ for $p \in \mathcal{H})$, and let $Z$ be any vector field on ${ }^{4} M$ which is transverse to $\mathcal{H}$. There exists a neighborhood $\mathcal{O}=\mathcal{O}(Z, \mathcal{H})$ on which $\nabla_{Z} \varphi$ is nowhere vanishing. Without loss of generality, we shall assume that $\nabla_{Z} \varphi>0$ on $\mathcal{O}(Z, \varphi)$. We will also assume (as an extra restriction), that

$$
\left.\nabla^{\mu} \varphi \nabla_{\mu} \varphi\right|_{\mathcal{O} \cap \dot{\mathcal{D}}(\Sigma)}<0 .
$$

Here $\stackrel{\circ}{\mathcal{D}}(\Sigma)$ indicates the globally hyperbolic region ${ }^{4}$ of $\left({ }^{4} M, g\right)$ - with Cauchy surface $\Sigma$ - to the past of $\mathcal{H}$. Note that $\Sigma$ is necessarily diffeomorphic to $\mathcal{H}$ [Ch-I2].

On $\mathcal{O}$, we consider the $Z$ and $\varphi$ dependent one-form

$$
\omega(Z, \varphi):=\frac{\nabla_{Z}\left(\nabla^{\mu} \varphi \nabla_{\mu} \varphi\right) g(Z,)}{\left(\nabla_{Z} \varphi\right)^{2}} .
$$

Then for $p \epsilon^{2} M$, let us define the function

$$
\beta(p, \varphi):=\int_{\Gamma(p)} \omega(Z, \varphi)
$$

${ }^{4}$ More specifically, $\stackrel{\circ}{\mathcal{D}}(\Sigma)$ is the interior of $\mathcal{D}(\Sigma)$, the domain of dependence of $\Sigma$. 
We claim that the right-hand side of equation (7) - and hence the function $\beta-$ is independent of the choice of vector field $Z$. To see this, we first note that for any nowhere vanishing function $\chi$ on $\mathcal{O}$, we have from equation (6) that

$$
\omega(\chi Z, \varphi)=\omega(Z, \varphi)
$$

thus $\omega$ and consequently $\beta$ are independent of the scale of $Z$. Next, say that $\tilde{Z}$ is any other vector field which is transverse to $\mathcal{H}$. There exists a positive function $\chi$ on $\mathcal{O}$ and a vector field $Y$ on $\mathcal{O}$, with $Y$ tangent to $\mathcal{H}$, such that $\tilde{Z}=\chi Z+Y$. Since $\varphi$ is a defining function of $\mathcal{H}$ and since $\mathcal{H}$ is null, one has $\left.\varphi\right|_{\mathcal{H}}=0$ and $\left.\nabla^{\mu} \varphi \nabla_{\mu} \varphi\right|_{\mathcal{H}}=0$, so $\left.\nabla_{\tilde{Z}} \varphi\right|_{\mathcal{H}}=\left.\nabla_{\chi Z} \varphi\right|_{\mathcal{H}}$ and $\left.\nabla_{\tilde{Z}}\left(\nabla^{\mu} \varphi \nabla_{\mu} \varphi\right)\right|_{\mathcal{H}}=\left.\nabla_{\chi Z}\left(\nabla^{\mu} \varphi \nabla_{\mu} \varphi\right)\right|_{\mathcal{H}}$. It also follows from the nullness of $\mathcal{H}$ that if we let $\frac{d}{d s}$ denote the tangent vector to $\Gamma(p)$, then

$$
\left.\left.g\left(\tilde{Z}, \frac{d}{d s}\right)\right|_{\mathcal{H}} \propto \nabla_{\tilde{Z}} \varphi\right|_{\mathcal{H}}=\left.\nabla_{\chi Z} \varphi\right|_{\mathcal{H}}
$$

Hence we get the same value for $\beta$ for any choice of vector field $Z$ which is transverse to $\mathcal{H}$.

We now wish to analyze the dependence of $\beta$ upon the choice of the defining function $\varphi$. So let $\varphi$ and $\tilde{\varphi}$ be two different defining functions for $\mathcal{H}$. Passing to a subset of $\mathcal{O}$ if necessary, we find that there exists a positive function $\mu$ on $\mathcal{O}$ such that $\tilde{\varphi}=\mu \varphi$. We then calculate, at $\mathcal{H}$,

$$
\omega(Z, \tilde{\varphi})=\omega(Z, \varphi)+\frac{2 \nabla^{\alpha} \varphi \nabla_{\alpha}(\ln \mu)}{\nabla_{Z} \varphi} g(Z,) .
$$

We may choose coordinates $\left(s, x^{a}\right)$ on $\mathcal{H}$ in a neighborhood of $p$ so that $g^{-1}(d \varphi)=,\frac{\partial}{\partial s}$, with $\frac{\partial}{\partial s}$ tangent to the generators of $\mathcal{H}$. Then a straightforward calculation gives

$$
\beta(p, \tilde{\varphi})=\beta(p, \varphi)+2 \ell n\left(\frac{\mu(\Psi(p))}{\mu(p)}\right) .
$$

It follows that unless $\Psi(p)=p$ for all $p \in{ }^{2} M, \beta$ depends on the choice of the defining function $\varphi$.

To remove this dependence, we set

$$
Q:=\sup _{\varphi} \inf _{p \in^{2} M} \beta(p, \varphi),
$$

where the sup is taken over all smooth defining functions $\varphi$ defined on some $\varphi$-dependent neighborhood of $\mathcal{H}$. This is the quantity we will use in stating sufficient conditions for horizon stability. 
It would be very convenient if $Q$ were infinite for every spacetime with a Cauchy horizon. This, however, is not the case. In fact, one finds the following:

Proposition 1. Let $\varphi$ be any defining function for $\mathcal{H}$. One has

$$
Q \leq \sup _{p \in \in^{2} M} \beta(p, \varphi)
$$

Proof. Suppose first that the Poincare map $\Psi$ is the identity. It then follows that $\beta$ is $\varphi$-independent, as seen from equation (10), and thus (12) is obvious.

If $\Psi$ is not the identity, then for any $p \in{ }^{2} M$, equation (10) implies that for repeated iterations of the map $\Psi$, one has

$$
\frac{1}{n+1} \sum_{i=0}^{n} \beta\left(\Psi^{i}(p), \mu \varphi\right)=\frac{1}{n+1} \sum_{i=0}^{n} \beta\left(\Psi^{i}(p), \varphi\right)+\frac{2}{n+1} \ln \left(\frac{\mu\left(\Psi^{n}(p)\right)}{\mu(p)}\right) .
$$

If there is some point $p_{*} \in{ }^{2} M$ such that $\psi^{n}\left(p_{*}\right)=p_{*}$ for some $n$, then (12) follows from (13), evaluated at $p=p_{*}$. If however no such point exists, then from equation (13) we calculate

$$
\inf _{p \in \in^{2} M} \beta(p, \mu \varphi) \leq \sup _{p \in \in^{2} M} \beta(p, \varphi)+\frac{2}{n+1} \ln \left(\frac{\max _{2 \mu}}{\min _{2 \mu}}\right) .
$$

Then letting $n \rightarrow \infty$ in (14), we obtain condition (12).

This proposition shows that $Q$ is always finite. Our main result will guarantee that the horizon is stable so long as $Q$ is greater than a certain quantity. This quantity involves the $C^{1}$ norm of the Poincaré map, which we write as $\|\Psi\|_{1}$, and define as follows: Fix some arbitrary Riemannian metric $h$ on ${ }^{2} M$, and let $d(p, q)$ denote the associated distance function. For any (Poincaré) map $\Psi:{ }^{2} M \rightarrow{ }^{2} M$, we set

$$
\|\Psi\|_{0}:=\sup _{p \in \in^{2} M} d(\Psi(p), p)
$$

and then

$$
\|\Psi\|_{1}=\|\Psi\|_{0}+|\Psi|_{1}
$$


where

$$
\begin{aligned}
|\Psi|_{1} & =\sup _{p \in{ }^{2} M}\left(\operatorname{tr}_{h} \Psi^{*} h\right)(p) \\
& =\sup _{p \in^{2} M} h^{i j}(p) h_{k l}(\Psi(p)) \frac{\partial \Psi^{k}}{\partial x^{i}}(p) \frac{\partial \Psi^{l}}{\partial x^{j}}(p) .
\end{aligned}
$$

Higher order norms $\|\Psi\|_{n}$ of $\Psi$ can be defined in a similar way, using appropriately defined covariant derivatives of $\Psi$.

To show that the inequality involving $Q$ and $\|\Psi\|_{1}$ in the hypothesis of our main result (stated below) can be achieved - and hence our result is not empty - we wish to show that the quantities $Q$ and $\|\Psi\|_{1}$ are independent in a certain sense (so long as no field equations are imposed). More specifically, we want to show that for a given compact submanifold $\mathcal{H}$ of ${ }^{4} M$, with a given set of orbits on $\mathcal{H}$ and a given return map $\Psi$, there exist many metrics $g$ on ${ }^{4} M$ for which $\mathcal{H}$ is a Cauchy horizon with those given orbits as null generators, and for which $Q$ takes on arbitrarily large values.

To see this, consider ${ }^{4} M, \mathcal{H}$, and $\Psi$ as just described. As shown in [Ch-I1], one can find a metric $g$ for which $\mathcal{H}$ is a Cauchy horizon and for which $\Psi$ is the Poincare map of the flow of the generators on $\mathcal{H}$. Generally $Q$ may be small. Let us now modify $g$ so that $Q$ becomes arbitrarily large: We need (i) a $C^{2}$ defining function $\varphi$ for $\mathcal{H}$, (ii) a $C^{2}$ timelike vector field $T$, and (iii) a $C^{2}$ non-negative function $\nu$ which is equal to one in a neighborhood $U$ of $\mathcal{H}$, and zero outside of some larger neighborhood $V \supset U$. We claim that for all positive constants $\lambda$, the metrics $\tilde{g}$ defined by the equation

$$
\underset{(\lambda)}{\tilde{g}_{\alpha \beta}}:=g_{\alpha \beta}-\lambda \varphi \nu T_{\alpha} T_{\beta}
$$

are spacetime metrics on ${ }^{4} M$, with $\mathcal{H}$ a Cauchy horizon for each spacetime $\left(\begin{array}{c}{ }^{4} M, \underset{(\lambda)}{\tilde{g}} \\ { }_{(\lambda)}\end{array}\right)$. To verify this, note that every timelike vector field in $\left({ }^{4} M, g\right)$ remains timelike in all $\left({ }^{4} M, \underset{(\lambda)}{\tilde{g}}\right)$, note that $\mathcal{H}$ is a null hypersurface in all $\left({ }^{4} M \underset{(\lambda)}{\underset{g}{\tilde{g}}}\right)$, and note that any time function defined on the globally hyperbolic region $\stackrel{\circ}{\mathcal{D}}(\Sigma)$ of $\left({ }^{4} M, g\right)$ is a time function in all $\left(\stackrel{\circ}{\mathcal{D}}(\Sigma),\left.\underset{(\lambda)}{\tilde{g}}\right|_{\stackrel{\circ}{\mathcal{D}}(\Sigma)}\right)$. 
We also claim that as $\lambda$ increases, $Q$ increases as well. Indeed, it follows from equations (6), (7), (11) and (15) that $\lim _{\lambda \rightarrow \infty} Q \tilde{g}=+\infty$. Meanwhile,

since $\|\Psi\|_{1}$ involves only the metric on $\mathcal{H}$ and since $\left.\underset{(\lambda)}{\tilde{g}}\right|_{\mathcal{H}}=\left.\tilde{g}\right|_{\mathcal{H}},\|\psi\|_{1}$ is unaffected by changes in $\lambda$. So, we see that the inequality involving $Q$ and $\|\Psi\|_{1}$ can be achieved by construction (so long as no field equations are imposed).

We now state our main result:

Theorem 2. There exist constants $k_{1}$ and $k_{2}$ such that the following holds: Let ${ }^{2} M$ be a two-dimensional manifold and let $\Psi:{ }^{2} M \rightarrow{ }^{2} M$ be a $C^{k}$ diffeomorphism, for $k \geq \operatorname{Max}\left\{k_{1}, k_{2}\right\}$. Consider any spacetime $\left({ }^{4} M, \hat{g}\right)$ with a $C^{k-1}$ metric and with a compact embedded $C^{k}$ Cauchy horizon $\hat{\mathcal{H}}$ admitting a section with Poincaré map $\Psi$. There exists a constant $\kappa\left(k,\|\hat{\Psi}\|_{1}\right)$ such that if $Q \geq \kappa$, then there is a differentiable map

$$
L_{(k)}: g \rightarrow \mathcal{H}
$$

which assigns, to every $C^{k-1}$ metric $g$ in a neighborhood $\mathcal{O}_{(k-1)} \subset C^{k-1}\left({ }^{4} M\right)$ of $\hat{g}$, a Cauchy horizon $\mathcal{H}$ for the spacetime $\left({ }^{4} M, g\right)$, with $\mathcal{H}$ a compact embedded $C^{k-k_{2}}$ submanifold of ${ }^{4} M$.

\section{Remarks.}

1) The neighborhood $\mathcal{O}_{(k-1)}$ of metrics $g$ near $\hat{g}$ may be characterized as follows: For some conditionally compact neighborhood $U$ of $\hat{\mathcal{H}}$ in ${ }^{4} M$, there exists some $\varepsilon>0$ such that

$$
\mathcal{O}_{(k-1)}=\left\{g \mid\|g-\hat{g}\|_{C^{k-1}(\bar{U})}<\varepsilon\right\} .
$$

2) One may verify that the example given in $\S 2$ of a spacetime with an unstable Cauchy horizon fails to satisfy the hypothesis of this theorem. In particular, one finds that $Q$ for this spacetime is zero, while $\kappa$ is positive.

3) The condition (6) $\left.\nabla^{\mu} \varphi \nabla_{\mu} \varphi\right|_{\mathcal{O} \cap \mathcal{D}(\Sigma)}<0$ cited as a needed restriction for proving stability is implicit in the inequality $Q \geq \kappa$.

4) This theorem, together with our results elsewhere [Ch-I1], implies "topological stability" of $\hat{\mathcal{H}}$ as well as "locational stability" of $\hat{\mathcal{H}}$ in the sense of Beem [Be].

5) In recent studies of the behavior of chronology horizons and the "Fountain Conjecture" [Haw3], [Th], [Ch-I1], the question of the stability of certain global properties of the flow of generators of a Cauchy horizon has been 
raised. A result relevant to these studies can be inferred from Theorem 2 and its proof as follows: As we show in the proof below, under appropriate conditions, a defining function $\varphi$ of the horizon $\mathcal{H}$ is a differentiable function of the metric $g$. Moreover, if $Q \geq \kappa\left(k=\operatorname{Max}\left(k_{1}, k_{2}+1\right)\right.$ then $\nabla \varphi$ is a differentiable function of $g$ as well. Since $\nabla \varphi$ is tangent to the generators of $\mathcal{H}$, the flow of these generators is also a differentiable function of the metric $g$, so long as the dynamics of the generators of $\hat{\mathcal{H}}$ is structurally stable, and so long as $Q \geq \kappa\left(k=\operatorname{Max}\left(k_{1}, k_{2}+2\right)\right.$.

6) The proof below suggests very strongly (but fails to prove) that for any $k \geq 1$, if $Q$ is smaller than $\kappa\left(k,\|\Psi\|_{1}\right)$, then generic perturbations of the metric, no matter how small, either destroy the horizon or lead to a horizon which is not of differentiability class $C^{k}$. We present a result supporting this expectation (in the case $k=1$ ) in $\S 4$ below.

Proof of the theorem. The idea of the proof is to use an implicit function theorem type argument to construct the map we will call $L_{(k)}: g \mapsto \mathcal{H}(g)$. While we have not been able to find a direct formulation of this problem in terms of Banach spaces, we have been able to carry through this idea using the machinery of the Nash-Moser Theorem. In showing that the Nash-Moser Theorem works here, we shall use the terminology of [Ham], although the specific version of Nash-Moser we apply is that presented in [Hor1]. Note that we use the letter $c$ to denote a generic constant. (Its value may vary from line to line.)

Let $U$ be a neighborhood of $\hat{\mathcal{H}}$ which is diffeomorphic to $(-2 \varepsilon, 2 \varepsilon) \times \hat{\mathcal{H}}$ for some $\varepsilon>0$, and let $\left(x^{o}, x^{i}\right)$ be any coordinate system on $U$ such that $\hat{\mathcal{H}}=\left\{p \in U \mid x^{o}(p)=0\right\}$ and such that the set $U_{-}:=\left\{p \in U \mid x^{o}(p)<0\right\}$ satisfies the condition $U_{-} \subset \stackrel{\circ}{\mathcal{D}}(\Sigma)$, where $\Sigma$ is a partial Cauchy surface for the globally hyperbolic region of $\left({ }^{4} M, \hat{g}\right)$. So long as $Q>0$, we may choose $x^{0}$ and $\varepsilon$ so that $x^{0}$ is a time function on $(-\varepsilon, 0) \times \hat{\mathcal{H}}$. Since $x^{0}$ is a defining function of $\hat{\mathcal{H}}$, note that we have, from Proposition $1, \sup _{p \in \in^{2} M} \beta\left(p, x^{0}\right) \geq Q$. Note also that the vector field

$$
Z=Z^{u} \partial_{u}:=g^{\mu \nu} x^{0}{ }_{\nu} \partial_{\mu}
$$

is tangent to the generators of $\hat{\mathcal{H}}$.

Now, let $\mathcal{M}^{k-1}$ be the space of $C^{k-1}$ Lorentz metrics on $[-\varepsilon,+\varepsilon] \times \hat{\mathcal{H}}$. We consider the map

$$
F: \mathcal{M}^{k-1} \times C^{k}(\hat{\mathcal{H}}) \rightarrow C^{k-1}(\hat{\mathcal{H}})
$$

$$
(g, f) \mapsto g^{\mu \nu}\left(x^{0}=-f\left(x^{i}\right), x^{i}\right)\left(x^{0}+f\left(x^{i}\right)\right)_{, \mu}\left(x^{0}+f\left(x^{k}\right)\right)_{, \nu} .
$$


For any given metric $g \in \mathcal{M}^{k-1}$, we claim that if there is a corresponding function $\theta_{g} \in C^{k}: \hat{\mathcal{H}} \rightarrow \mathbb{R}$ such that $F\left(g, \theta_{g}\right)=0$, and if the $C^{2}(\hat{\mathcal{H}})$ norm of $\theta_{g}$ is small enough, then the set

$$
\mathcal{H}:=\left\{p \in[-\varepsilon, \varepsilon] \times \hat{\mathcal{H}} \mid x^{0}(p)+\theta_{g}(p)=0\right\}
$$

is a Cauchy horizon in $\left({ }^{4} M, g\right)$. Indeed, so long as $\left\|\theta_{g}\right\|_{C^{1}(\hat{\mathcal{H}})}$ is small enough, one has that $\mathcal{H}$ from (19) is a $C^{1}$ submanifold of ${ }^{4} M$. Moreover, we also have that for $\left\|\theta_{g}\right\|_{C^{2}(\hat{\mathcal{H}})}$ small enough,

$$
\left.\frac{\partial}{\partial x^{0}}\left[g^{\mu \nu}\left(x^{0}+\theta_{g}\right),{ }_{\mu}\left(x^{0}+\theta_{g}\right),{ }_{\nu}\right]\right|_{\mathcal{H}}>0
$$

as a consequence of the fact that $\hat{\mathcal{H}}$ is compact, and the fact that $U_{-}$is globally hyperbolic with $x^{0}$ serving as a time function for $U_{-}$. Thus $x^{0}+\theta_{g}$ is a time function for the region in which $x^{0}+\theta_{g}<0$; and consequently $\mathcal{H}$ is a Cauchy horizon of the same differentiability class as $\theta_{g}$.

One would be tempted to use the implicit function theorem to prove the existence of the map $\theta_{g}$. The problem, however, is that while for a fixed $C^{k-1}$ metric the map $F(g$,$) maps C^{k}$ functions to $C^{k-1}$ functions, the map which is the inverse of $D_{2} F(g$,$) maps C^{k-1}$ functions to functions which are not generally $C^{k}$. Thus, the standard implicit function theorem does not seem to be able to handle our problem. We shall show that we can use Hörmander's version of the Nash-Moser theorem to obtain the map $\theta_{g}$ discussed above.

Let $\bar{X}$ be the tame Fréchet space of smooth Lorentz metrics on $[-\in, \in] \times \hat{\mathcal{H}}$ (See Corollaries II.1.3.7 and II.1.3.9 in [Ham]) and let $\bar{Y}$ be the tame Fréchet space of smooth functions $f: \hat{\mathcal{H}} \rightarrow R$ which satisfy $|f|<\varepsilon$. (See Theorem II.1.3.6 in [Ham].) With a small abuse of notation, let us denote by $F$ the map from $\bar{X} \times \bar{Y}$ to $\bar{Y}$ obtained by restricting $F$ from (18) to the domain $\bar{X} \times \bar{Y}$.

From what is proven in Section II.2 of [Ham], it is readily seen that $F$ as defined above by equation (18) is a smooth tame map. Then to apply the Nash-Moser Theorem to this map we need to do two things:

1) Show that for all $g$ in some neighborhood of $\hat{g}$, the map $D_{2} F$ - which is the partial derivative of $F$ with respect to its second slot - has a "right inverse" in a sense which is made precise in Proposition 3.

2) Obtain appropriate estimates for this "right inverse" of $D_{2} F$. 
Now, the derivative $D_{2} F$ of the map $F$, when acting on a function $\alpha \in$ $C^{\infty}(\hat{\mathcal{H}})$, is given by

$$
D_{2} F(g, f) \alpha=2 g^{\mu b}\left(x^{0}=-f\left(x^{i}\right), x^{i}\right)\left(x^{0}+f\left(x^{i}\right)\right),{ }_{\mu} \frac{\partial \alpha}{\partial x^{b}}
$$

The equation

$$
-\frac{\partial g^{\mu \nu}}{\partial x^{0}}\left(x^{0}=-f\left(x^{i}\right), x^{i}\right)\left(x^{0}+f\left(x^{i}\right)\right),{ }_{\mu}\left(x^{0}+f\left(x^{i}\right)\right), \nu \alpha .
$$

can thus be written in the form

$$
Y(\alpha)+\gamma \alpha=\mu
$$

where the vector field $Y$ is given (in component form) by

$$
\begin{aligned}
Y^{b}\left(x^{j}\right) & =Y^{b}[g, f]\left(x^{j}\right) \\
& =2 g^{\mu b}\left(x^{0}=-f\left(x^{j}\right), x^{i}\right)\left(x^{0}+f\left(x^{i}\right)\right), \mu
\end{aligned}
$$

and the function $\gamma$ is given by

$$
\begin{aligned}
\gamma\left(x^{j}\right) & =\gamma[g, f]\left(x^{j}\right) \\
& =-\frac{\partial g^{\mu \nu}}{\partial x^{0}}\left(x^{0}=-f\left(x^{j}\right), x^{i}\right)\left(x^{0}+f\left(x^{i}\right),{ }_{\mu}\left(x^{0}+f\left(x^{i}\right)\right),{ }_{\nu}\right.
\end{aligned}
$$

Note that for small $f, \gamma$ is negative.

From equation (24), it follows that there exists a neighborhood $\mathcal{O} \subset$ $\mathcal{M}^{k-1} \times C^{k}(\hat{\mathcal{H}})$ of $(\hat{g}, 0) \in \mathcal{M}^{k-1} \times C^{k}(\hat{\mathcal{H}})$, of the form $\mathcal{O}=\left\{\|g-\hat{g}\|_{C^{1}}+\right.$ $\left.\|f\|_{C^{1}}<\varepsilon_{1}\right\}$ for some $\varepsilon_{1}$, such that for all $(g, f) \in \mathcal{O}$, the manifold ${ }^{2} M$ is a global cross-section for the flow of $Y=Y^{b} \frac{\partial}{\partial x^{6}}$ on $\hat{\mathcal{H}}$, with Poincaré map $\Psi[g, f]$. Now let $\xi_{s}[Y](p)$ denote the flow of $Y$ on $\hat{\mathcal{H}}$ through a point $p \in{ }^{2} M$, and for each such $p$ let $t(p)$ be the smallest strictly positive number such that $\xi_{t(p)}[Y](p)$ is contained in ${ }^{2} M$. Replacing $\mathcal{O}$ by a subset thereof if necessary, we find for all $(g, f) \in \mathcal{O}$ that

$$
\int_{0}^{t(p)} \gamma\left(\xi_{s}[Y](p)\right) d s<-\frac{Q}{2} .
$$

Thus we may now prove the following.

Proposition 3. Suppose that $Q>0$. For all $(g, f) \in \mathcal{O}$, and for all $\mu \in$ $C^{\infty}(\hat{\mathcal{H}})$, the equation (23) has a unique solution $\alpha \in C^{0}(\hat{\mathcal{H}})$. [We shall use $\ell_{[g, f]}$ to denote the map from $\mu$ to the solution $\alpha$.] 
Proof of Proposition 3. Consider the map

$$
\begin{aligned}
\psi:[0,1] \times{ }^{2} M & \rightarrow \hat{\mathcal{H}} \\
(s, p) & \mapsto \xi_{s t(p)}[Y](p) .
\end{aligned}
$$

One verifies that $\psi$ is locally invertible on $[0,1] \times{ }^{2} M$, and that its restriction to $(0,1) \times{ }^{2} M$ is a $C^{k-1}$ diffeomorphism between $(0,1) \times{ }^{2} M$ and $\hat{\mathcal{H}} \backslash{ }^{2} M$. Moreover, $\psi(1$,$) coincides with the Poincaré map \Psi$ of the flow of $Y$.

Let us define

$$
\begin{aligned}
& \hat{\alpha}:=\alpha \circ \psi, \\
& \hat{\gamma}:=\gamma \circ \psi,
\end{aligned}
$$

and

$$
\hat{\mu}:=\mu \circ \psi \text {. }
$$

Then equation (23) on $\hat{\mathcal{H}}$ is equivalent to the following boundary value problem on $[0,1] \times{ }^{2} M$ :

$$
\begin{gathered}
\frac{d}{d s} \hat{\alpha}+\hat{\gamma} \hat{\alpha}=\mu \\
\hat{\alpha}(1, p)=\hat{\alpha}(0, \Psi(p))
\end{gathered}
$$

One readily solves equation (29) for $\hat{\alpha}(s, p)$ in terms of $\hat{\alpha}(0, p)$; the solution takes the form

$$
\hat{\alpha}(s, p)=\exp \left[-\int_{0}^{s} \hat{\gamma}(t, p) d t\right]\left\{\hat{\alpha}(0, p)+\int_{0}^{s} \hat{\mu}(t, p) \exp \left[\int_{0}^{t} \hat{\gamma}(u, p) d u\right]\right\}
$$

To take account of the boundary condition (30), we combine equation (31) with equation (30) to obtain

$$
A=(A \circ \Psi) G+H
$$

where

$$
\begin{aligned}
A(p) & :=\hat{\alpha}(0, p) \\
G(p) & :=\exp \left[\int_{0}^{1} \hat{\gamma}(t, p) d t\right] \\
H(p) & :=-\int_{0}^{1} \hat{\mu}(t, p) \exp \left[\int_{0}^{1} \hat{\gamma}(u, p) d u\right] d t
\end{aligned}
$$


and $\Psi$ is the Poincaré map, as before.

We claim that there is a unique function $A(p)$ which satisfies equation (32) and that it may be expressed as

$$
A=H_{0}+G_{0} H_{1}+\sum_{i=1}^{\infty} G_{0} \cdots G_{i-1} H_{i},
$$

where the subscript notation refers to the following convention: For any $f \in C^{\infty}\left({ }^{2} M\right)$, we define $f_{0}:=f, f_{1}:=f \circ \psi, \ldots, f_{j}:=f_{j-1} \circ \psi$. To check this claim, we first note that since $\hat{\gamma}$ [see equation (25)] is negative, $G(p)<1$. It follows from this, and from the compactness of ${ }^{2} M$, that the series (34) converges absolutely. One verifies by substitution that $A$ from (34) satisfies (32). Then to prove uniqueness, we consider any pair of solutions $A$ and $\tilde{A}$ of (32). One finds that

$$
A-\tilde{A}=\left(A_{1}-\tilde{A}_{1}\right) G
$$

so it follows that

$$
\begin{aligned}
\sup _{{ }_{2}}\left(A_{1}-\tilde{A}_{1}\right) & \leq \sup _{2 M}\left[\left(A_{1}-\tilde{A}_{1}\right) \circ \psi\right] \sup _{{ }_{2}} G \\
& =\sup _{2 M}\left(A_{1}-\tilde{A}_{1}\right) \sup _{2 M} G .
\end{aligned}
$$

Since $\sup _{2}{ }_{M} G<1$, we must have $A=\tilde{A}$.

Now that we have an explicit expression for $\hat{\alpha}(0, p)=A(p)$, we may substitute it into (31); we thereby obtain a (unique) solution to the boundary value problem (29) - (30). Because $\psi$ is locally invertible, we may set $\alpha:=$ $\hat{\alpha} \circ \psi^{-1}$, and we verify that this function solves equation (23) on $\hat{\mathcal{H}}$. We then set $\ell_{[g, f]} \mu=\alpha$.

This proposition establishes the existence, in a certain sense, of a right inverse of $D_{2} F$ for $g$ near $\hat{g}$. We now need to establish certain estimates for this right inverse, which (as in Proposition 3) we represent as $\ell_{[g, f]}$. To obtain these estimates, we first need a few intermediate results, which we prove as a sequence of lemmas.

Lemma 4. Let $\left\{\alpha_{i} \mid i=1, \ldots, I\right\}$ be a set of strictly positive constants, with $\sum_{i=1}^{I} \alpha_{i}=1$. For any pair of sets of constants $\left\{b_{i} \mid i=1, \ldots, I\right\}$ and 
$\left\{d_{i} \mid i=1, \ldots, I\right\}$ which satisfy the condition $b_{i} d_{i} \geq 0$, there exists a constant $c\left(\alpha_{i}\right)$ such that

$$
\prod_{i=1}^{I} b_{i}^{\alpha_{i}} d_{i}^{1-\alpha_{i}} \leq c\left(\alpha_{i}\right) \sum_{i=1}^{I}\left(b_{i} / d_{i}\right)\left(d_{1} d_{2} \cdots d_{I}\right)
$$

(Whenever any of the $d_{i}$ 's vanish, (37) is to be understood in the obvious way.)

Proof of Lemma 4. Since equation (37) is invariant under the transformation

$$
b_{i} \rightarrow \lambda \lambda_{i} b_{i} \quad d_{i} \rightarrow \lambda \lambda_{i} d_{i}
$$

for any $\lambda>0$ and $\lambda_{i}>0$, it is sufficient to verify (37) for $d_{i}=1$, and $\sum_{i=1}^{I} b_{i}=1$. But then the resulting condition - that there exists $c\left(\alpha_{i}\right)$ such that $\prod_{i=1}^{I} b_{i}^{\alpha_{i}} \leq c\left(\alpha_{i}\right)$ - follows from the continuity of the function $F\left(b_{i}\right)=\prod_{i=1}^{I} b^{\alpha_{i}}$ on the compact set $\left\{b_{i} \mid b_{i} \geq 0\right.$ and $\left.\Sigma b_{i}=1\right\}$.

We use Lemma 4 primarily to prove the following:

Lemma 5. Let $\left\{f_{i} \mid i=1, \ldots, I\right\}$ be a set of functions in $C^{n}\left({ }^{2} M\right)$. There exists a constant $c(n)$ such that

$$
\left\|f_{1} \cdots f_{I}\right\|_{C^{n}(2 M)} \leq c(n) \sum_{i=1}^{I} \frac{\left\|f_{i}\right\|_{C^{n}(2 M)}}{\left\|f_{i}\right\|_{C^{0}(2 M)}}\left\|f_{1}\right\|_{C^{0}(2 M)} \cdots\left\|f_{I}\right\|_{C^{0}(2 M)} .
$$

Proof of Lemma 5. Let us consider the $m$-th order derivatives, for $1 \leq$ $m \leq n$, which are included in the norm \|\|$_{\left.C^{n}{ }^{2} M\right)}$. Hence we consider multiindices $\alpha=\left(\alpha_{1}, \alpha_{2}\right)$ for ${ }^{2} M$ summing to $n$. As a consequence of the product rule, for each such $\alpha$ we have

$$
\left|\partial^{\alpha}\left(f_{1} \cdots f_{I}\right)\right|=\left|\sum_{\beta^{1}+\cdots \beta^{I}=\alpha}\left(\partial^{\beta^{1}} f_{1} \cdots \partial^{\beta^{I}} f_{I}\right)\right|,
$$

where the sum is over all sets of $I$ multi-indices $\beta^{1}=\left(\beta_{1}^{1}, \beta_{2}^{1}\right), \cdots \beta^{I}=$ $\left(\beta_{1}^{I}, \beta_{2}^{I}\right)$, such that $\sum_{j=1}^{I} \beta_{1}^{j}=\alpha_{1}$ and $\sum_{j=1}^{I} \beta_{2}^{j}=\alpha_{2}$. It now follows from 
interpolation arguments (see, e.g. Theorem A.5 in [Hor1]) applied to the right hand side of equation (40), that for some constant $\tilde{c}(n)$,

$$
\begin{aligned}
& \left|\partial^{\alpha}\left(f_{1} \cdots f_{I}\right)\right| \\
& \quad \leq \tilde{c}(n) \sum_{\beta^{1}+\cdots \beta^{\prime}=\alpha}\left\|f_{1}\right\|_{C^{n}(2 M)}^{\frac{\left|\beta^{1}\right|}{n}}\left\|f_{1}\right\|_{C^{0}\left(2^{2} M\right)}^{1-\frac{\left|\beta^{1}\right|}{n}} \cdots\left\|f_{I}\right\|_{C^{n}(2 M)}^{\frac{\left|\beta^{I}\right|}{n}}\left\|f_{I}\right\|_{C^{0}\left(2^{2} M\right)}^{1-\frac{\left|\beta^{I}\right|}{n}}
\end{aligned}
$$

If we now apply Lemma 4 , with $b_{i}=\|f\|_{C^{n}\left({ }^{2} M\right)}, \alpha_{i}=\frac{\left|\beta^{i}\right|}{n}$, and $d_{i}=$ $\left\|f_{i}\right\|_{C^{0}\left({ }^{2} M\right)}$, and if we then sum the results over all $m$ for which $1 \leq m \leq n$, then the result follows.

The next lemma involves the effect of diffeomorphisms on norms of functions. So let $\operatorname{Diff}_{C^{n}}(M)$ denote the group of those diffeomorphisms of a manifold $M$ which are of differentiability class $C^{n}$. We have the following:

Lemma 6. Let $\psi \in \operatorname{Diff}_{C^{n}}(M)$, with $n \geq 1$ and with $\|\psi\|_{C^{1}(M)} \leq \zeta$ for some constant $\zeta$. Let $f \in C^{n}(M)$, and define the sequence $\left\{f_{j}\right\} \in C^{n}(M)$ by $f_{0}:=f, f_{1}:=f \circ \psi, \cdots f_{j+1}:=f_{j} \circ \psi$. There exists a constant $B(n, \zeta)$ such that

$$
\left\|f_{j}\right\|_{C^{n}(M)} \leq(B(n, \zeta))^{j}\left(\|f\|_{C^{n}(M)}+\|f\|_{C^{1}(M)}\|\psi\|_{C^{n}(M)}\right) .
$$

Proof of Lemma 6. We consider first the special case in which $n=1$. Then one calculates (using the shorthand \|\|$_{n}:=\|\|_{C^{n}(M)}$ here and below in the proof of this lemma), for any $i$,

$$
\begin{aligned}
\left\|\partial f_{i}\right\|_{0} & =\left\|(\partial f)_{i}(\partial \psi)_{i-1} \cdots(\partial \psi)_{1}(\partial \psi)\right\|_{0} \\
& \leq\|\partial f\|_{0}\left(\|\partial \psi\|_{0}\right)^{i}
\end{aligned}
$$

Here, following the convention for $f_{i}$, we have $(\partial f)_{i}:=\partial f \circ \psi{ }_{i} \psi \psi(\partial \psi)_{i-1}=$ $\partial \psi \circ \psi \circ \underset{i-1}{\ldots} \circ \psi$, etc. From equation (43) and the definition of the $C^{1}$ norm, we find

$$
\begin{aligned}
\left\|f_{i}\right\|_{1} & =\|f\|_{0}+\left\|\partial f_{i}\right\|_{0} \\
& \leq\left(1+\zeta^{i}\right)\|f\|_{1}
\end{aligned}
$$

which is consistent with (42). 
We next show that there exist constants $a_{i}(n, \zeta)$ and $b_{i}(n, \zeta)$ such that

$$
\left\|f_{i}\right\|_{n} \leq a_{i}(n, \zeta)\|f\|_{n}+b_{i}(n, \zeta)\|f\|_{1}\|\psi\|_{n} .
$$

We do this via induction on $i$, using the inequality (for some constant $d(n)$ )

$$
\|f \circ g\|_{n} \leq d(n)\left(\|f\|_{n}\|g\|_{1}^{n}+\|f\|_{1}\|g\|_{n}+\|f\|_{0}\right),
$$

which is proven in [Hor2] - see Theorem 3.6 - as well as in [Ham]. For $i=1$, inequality (45) follows from (46) if we choose $a_{1}=d(n)\left(1+\zeta^{n}\right)$ and $b_{1}=d(n)$. Now suppose that (45) holds for some $i \geq 1$. Inequality (46) then implies that

$$
\begin{aligned}
\left\|f_{i+1}\right\|_{n} & =\left\|f_{i} \circ \psi\right\|_{n} \\
& \leq d(n)\left[\left(1+\zeta^{n} a_{i}\right)\|f\|_{n}+\left(1+\zeta^{i}+\zeta^{n} b_{i}\right)\|f\|_{1}\|\psi\|_{n}\right]
\end{aligned}
$$

so if we can find $a_{i}(n, \zeta)$ such that

$$
a_{i+1} \leq d(n)\left(1+\zeta^{n} a_{i}\right)
$$

and $b_{i}(n, \zeta)$ such that

$$
b_{i+1} \leq d(n)\left(1+\zeta^{i}+\zeta^{n} b_{i}\right),
$$

then (45) holds for all values of $i$.

The recurrence inequalities (48) may be rewritten as

$$
a_{i+1} \leq c(n ; \zeta)\left(1+a_{i}\right)
$$

and

$$
b_{i+1} \leq c(n, \zeta)\left(1+\zeta^{i}+b_{i}\right)
$$

for appropriate $c(n, \zeta)$. From (49a), we calculate (assuming, without loss of generality, that $c(n, \zeta)>1)$

$$
\begin{aligned}
a_{i} & \leq c \sum_{\ell=0}^{i-2} c^{\ell}+c^{i-1} d(n)\left(1+\zeta^{n}\right) \\
& =\frac{c(n, \zeta)}{1-c(n, \zeta)}+\left[d(n)\left(1+\zeta^{n}\right)-\frac{c(n, \zeta)}{1-c(n, \zeta)}\right] c^{i-1}(n, \zeta) .
\end{aligned}
$$


Then from (49b) we find

$$
\begin{aligned}
b_{i} & \leq c+c \zeta^{i-1}+c b_{i-1} \\
& \leq c+c \zeta^{i-1}+c^{2}\left(1+\zeta^{i-2}+b_{i-2}\right) \\
& \leq c+c^{2}+\cdots+c^{i-1}+c \zeta^{i-1}+c^{2} \zeta^{i-2}+\cdots+c^{i-1} \zeta+c^{i-1} b_{1} \\
& =c \frac{\left(1-c^{i}\right)}{1-c}+c^{i-1}\left[\left(\frac{\zeta}{c}\right)^{i-1}+\cdots+\left(\frac{\zeta}{c}\right)\right]+d c^{i-1}
\end{aligned}
$$

$(50 \mathrm{~b})$

$$
=c\left(\frac{1-c^{i}}{1-c}\right)+\frac{\zeta}{c} \frac{c^{i}-\zeta^{i}}{c-\zeta}+\frac{d}{c} c^{i} .
$$

It is now clear, from (50a) and $(50 \mathrm{~b})$, that there is a constant $B(n, \zeta)$ such that $a_{i} \leq B^{i}$ and $b_{i} \leq B^{i}$. Inequality (42) is thus obtained, and so the lemma is verified.

We now use Lemmas 5 and 6 to derive an estimate which we will use to control $D_{2} F$, and to eventually show that its right inverse $\ell_{[g, f]}$ is tame, and is a composition of tame maps. We establish the estimate in the following:

Proposition 7. Consider the function

$$
A=H_{0}+G_{0} H_{1}+\sum_{j=2}^{\infty} G_{0} \cdots G_{j-1} H_{j}
$$

as in equation (34), where $H_{0}$ and $G_{0}$ are $C^{\ell}\left({ }^{2} M\right)$ functions, and where the indices on $H_{j}$ and $G_{k}$ refer to iterated compositions of $H_{0}$ and $G_{0}$ with a smooth diffeomorphism $\psi$, as in Proposition 3. For every integer $n \leq \ell$ there exists a positive $\varepsilon_{n}$ such that if $\|G\|_{0} \leq \varepsilon_{n}$, then $A \in C^{n}\left({ }^{2} M\right)$. As well, for each $i \leq n$, there exists a constant $c(i)$ such that

(52) $\|A\|_{C^{i}(2 M)}$

$\leq c(i)\left[\|H\|_{C^{i}(2 M)}+\|G\|_{C^{i}(2 M)}+\left(\|H\|_{C^{1}(2 M)}+\|G\|_{C^{1}\left({ }^{2} M\right)}\right)\|\psi\|_{C^{i}\left({ }^{2} M\right)}\right]$.

Proof of Proposition 7. We shall verify inequality (52) for the case $i=n$; the other cases are verified essentially the same way.

From (51) - and reverting to our notation \|\|$_{n} \leftrightarrow\|\|_{C^{n}\left({ }^{2} M\right)}$ - we have

$$
\|A\|_{n} \leq\left\|H_{0}\right\|_{n}+\left\|G_{0} H_{1}\right\|_{n}+\left\|\sum_{j=2}^{\infty} G_{0} \cdots G_{j-1} H_{j}\right\|_{n} .
$$


If we now apply Lemma 5 to each of the norms of products in (53), and if we recall that $\left\|H_{j}\right\|_{0}=\left\|H_{0}\right\|_{0}$ and $\left\|G_{j}\right\|_{0}=\left\|G_{0}\right\|_{0}=\sup _{2} M G$, then we have

$$
\|A\|_{n} \leq c(n)\left[\sum_{i=0}^{\infty} \mathcal{G}^{i}\left\|H_{i}\right\|_{n}+\left\|H_{0}\right\|_{0} \sum_{i=1}^{\infty} \sum_{j=0}^{i-1} \mathcal{G}^{i-1}\left\|G_{j}\right\|_{n}\right]
$$

where $\mathcal{G}:=\sup _{2} M G$, and $c(n)$ is some constant.

We may use Lemma 6 to obtain estimates for the terms $\left\|H_{i}\right\|_{n}$ and $\left\|G_{j}\right\|_{n}$ in (54). For the first of these, we have (for $\zeta:=\|\psi\|_{1}$ )

$$
\sum_{i=0}^{\infty} \mathcal{G}^{i}\left\|H_{i}\right\|_{n} \leq \sum_{i=0}^{\infty}(B(n, \zeta) \mathcal{G})^{i}\left(\left\|H_{0}\right\|_{n}+\left\|H_{0}\right\|_{1}\|\psi\|_{n}\right)
$$

We easily see that so long as

$$
B(n, \zeta) \mathcal{G}<1
$$

for all $n$ and $\zeta$, this series $\sum_{i=0}^{\infty} \mathcal{G}^{i}\left\|H_{i}\right\|_{n}$ converges. Applying Lemma 6 to $\left\|G_{j}\right\|_{n}$, we have

$$
\begin{aligned}
\sum_{i=1}^{\infty} \sum_{j=0}^{i-1} \mathcal{G}^{i-1}\left\|G_{j}\right\|_{n} & \leq \sum_{i=1}^{\infty} \mathcal{G}^{i-1} \sum_{j=0}^{i-1}(B(n, \zeta))^{j}\left(\|G\|_{n}+\|G\|_{1}\|\psi\|_{n}\right) \\
& =\sum_{i=1}^{\infty}(\mathcal{G} B(n, \zeta))^{i-1}\left(\frac{B-B^{1-i}}{B-1}\right)\left(\|G\|_{n}+\|G\|_{1}\|\psi\|_{n}\right) .
\end{aligned}
$$

This series, too, converges so long as inequality (56) holds for all $n$ and $\zeta$.

Estimates similar to those which produce (55) and (57) show that, so long as $B(n, \zeta) \mathcal{G}<1$, the sequence of partial sums from the right hand side of (51) is a Cauchy sequence in $C^{n}\left({ }^{2} M\right)$. Thus, if we set

$$
\varepsilon_{n}:=\frac{1}{B(n, \zeta)}
$$

and require that $\mathcal{G}=\|G\|_{0}<\varepsilon_{n}$, then the right hand side of equation (51) converges in $C^{n}\left({ }^{2} M\right)$. It follows that $A$ from equation (51) is a $C^{n}\left({ }^{2} M\right)$ function. Inequality (52), for $i=n$, immediately follows as well.

We need one more auxiliary result before we can proceed to finish proving our main result, Theorem 2 . 
Lemma 8. Let $h$ be a $C^{\infty}$ function on $\hat{\mathcal{H}}$, and let $Y$ be a $C^{\infty}$ vector field on $\hat{\mathcal{H}}$. Consider the map $\Lambda$, which to each such pair $(h, Y)$ assigns the $C^{\infty}$ function

$$
\begin{aligned}
\Lambda_{[h, Y]}:[0,1] \times \hat{\mathcal{H}} & \rightarrow \mathbb{R} \\
(t, p) & \mapsto h\left(\exp _{t Y}(p)\right),
\end{aligned}
$$

where "exp" denotes the exponential map on $\hat{\mathcal{H}}$. This map $\Lambda$ is tame.

Proof of Lemma 8. Since the composition of a pair of tame maps is tame [Ham], this lemma is proved ${ }^{5}$ if we can derive a tame estimate for solutions of the following initial value problem on $\mathbb{R}^{N}$

$$
\frac{d}{d t} x=H(x)
$$

and

$$
x(0)=\zeta .
$$

with $t \in[0, T]$ for some $T>0$. Here $H$ is a $C^{\infty}$ vector field on $\mathbb{R}^{N}$, and $\xi$ is a point in a ball $B_{r}$ of radius $r$ in $\mathbb{R}^{N}$.

Let $x$ be a solution of (60). We may view it as a $C^{\infty}$ function $x(t, \xi)$ of the pair $t \in[0, T]$ and $\xi \in B_{r}$. The estimate we seek takes the form of a set of inequalities for the norms $\|x\|_{C^{n}\left([0, T] \times B_{r}\right)}$. To obtain this estimate, it is useful to first consider the collection of $\mathbb{R}^{1}$-valued functions

$$
Z_{n}(t, \xi):=\sum_{0 \leq|\alpha| \leq n} e^{-\lambda t}\left|D_{2}^{\alpha}(x(t, \xi)-\xi)\right|^{2}
$$

where $\mathrm{n}$ is a positive integer, $\lambda$ is a constant to be chosen below, and $D_{2}^{\alpha} x$ indicates the multi-index $\alpha=\left(\alpha_{1}, \ldots \alpha_{N}\right)$ partial derivatives of $x$ with respect to its second slot (i.e., derivatives with respect to $\xi$ ). Making a specific choice for $\lambda$, we will derive an upper bound for the growth in $t$ of these $Z_{n}(t, \xi)$

\footnotetext{
${ }^{5}$ We use a partition of unity argument to go from the tame estimate for functions on $\mathbb{R}^{N}$, as obtained here, to a tame estimate for functions on $\hat{\mathcal{H}}$ (cf, the composition inequality (46)), as required for this lemma.

Note that the proof of this lemma essentially shows that the exponential map is a smooth tame map between the Fréchet space of $C^{\infty}$ fields on a compact manifold $M$ and the Fréchet space of smooth diffeomorphisms of $M$ (with the standard $C^{k}$ gradings).
} 
functions, and then use these bounds in obtaining the desired estimate for $x$. Note that for $n \geq 0$, one has

$$
Z_{n}(0, \xi)=0
$$

Calculating the time derivative of $Z_{n}(t, \xi)$ from its definition, we obtain

$$
\frac{\partial}{\partial t} Z_{n}=-\lambda Z_{n}+2 e^{-\lambda t} \sum_{0 \leq|\alpha| \leq n}\left\langle D_{2}^{\alpha}(x(t, \xi)-\xi) D_{2}^{\alpha} H\right\rangle .
$$

Then applying the Schwarz inequality to the sums of inner products in (63), we find

$$
\frac{\partial}{\partial t} Z_{n} \leq-\lambda Z_{n}+c(n, T) Z_{n}^{1 / 2}\|H \circ x\|_{C^{n}\left(B_{r}\right)}
$$

for some constant $c(n, T)$. To proceed from (64), we need to know something about $\|H \circ x\|_{C^{n}\left(B_{r}\right)}$. From the composition inequality (46), we find that

$$
\begin{aligned}
& \|H \circ x\|_{C^{n}\left(B_{r}\right)} \\
\leq & c(n)\left(\|H\|_{C^{n}\left(\mathbb{R}^{N}\right)}\|x\|_{C^{1}\left(B_{r}\right)}^{n}+\|H\|_{C^{1}\left(\mathbb{R}^{N}\right)}\|x\|_{C^{n}\left(B_{r}\right)}+\|H\|_{C^{0}\left(\mathbb{R}^{N}\right)}\right) .
\end{aligned}
$$

Then, since it follows from (60a) that there is a constant $c\left(T, n,\|H\|_{C^{1}\left(\mathbb{R}^{N}\right)}, r\right)$ such that

$$
\|x(t)\|_{C^{1}\left(B_{r}\right)} \leq c\left(T, n,\|H\|_{C^{1}\left(\mathbb{R}^{N}\right)}, r\right)
$$

for all $0 \leq t \leq T$, we may rewrite inequality (65) in the form

$$
\|H \circ x\|_{C^{n}\left(B_{r}\right)} \leq c\left(T, n,\|H\|_{C^{1}\left(\mathbb{R}^{N}\right)}, r\right)\left(\|x\|_{C^{n}\left(B_{r}\right)}+\|H\|_{C^{n}\left(\mathbb{R}^{N}\right)}\right),
$$

where the constant $c\left(T, n,\|H\|_{C^{1}\left(\mathbb{R}^{N}\right)}, r\right)$ is perhaps different in (67) and (66). We may now substitute this inequality for $\|H \circ x\|_{C^{n}\left(B_{r}\right)}$ into (64); we obtain (noting the relation between $Z_{n}^{1 / 2}$ and $\left(\|x\|_{C^{n}\left(B_{r}\right)}\right)$

$$
\begin{aligned}
& \frac{\partial}{\partial t} Z_{n} \leq-\lambda Z_{n}+c\left(T, n,\|H\|_{C^{1}\left(\mathbb{R}^{N}\right)}, r\right) \\
& \cdot\|H\|_{C^{n}\left(\mathbb{R}^{N}\right)} Z_{n}^{1 / 2}\left(Z_{n}^{1 / 2}+\|H\|_{C^{n}\left(\mathbb{R}^{N}\right)}+\|\operatorname{id}\|_{C^{n}\left(B_{r}\right)}\right)
\end{aligned}
$$

where id denotes the identity mapping on $B_{r}$. 
While (68) is not very useful for a general choice of $\lambda$, if we pick $\lambda=$ $c\left(T, n,\|H\|_{C^{1}\left(\mathbb{R}^{N}\right)}, r\right)$ then we have

$$
\frac{\partial}{\partial t} Z_{n} \leq c\left(T, n,\|H\|_{C^{1}\left(\mathbb{R}^{N}\right)}, r\right)\left(\|H\|_{C^{n}\left(\mathbb{R}^{N}\right)}+\| \text { id } \|_{C^{n}\left(B_{r}\right)}\right) Z_{n}^{1 / 2} .
$$

This easily integrates, and so for $n \geq 0$ (for which $Z_{n}(0, \xi)=0$, as noted above), we find

$$
Z_{n}^{1 / 2} \leq c\left(T, n,\|H\|_{C^{1}\left(\mathbb{R}^{N}\right)}, r\right)\left[\|H\|_{C^{n}\left(\mathbb{R}^{N}\right)} T / 2+1\right] .
$$

where again the constant $c$ is perhaps different in (69) and (70).

Based on these inequalities for $Z_{n}$, together with an analysis similar to that which we used to derive (70), we can show that for all $n>0$, and for all $t \in[0, T]$, there exists a constant $c\left(T, n,\|H\|_{C^{1}\left(\mathbb{R}^{N}\right)}, r\right)$ such that

$$
\|x(t)\|_{C^{n}\left(B_{r}\right)} \leq c\left(T, n,\|H\|_{C^{1}\left(\mathbb{R}^{N}\right)}, r\right)\left(\|H\|_{C^{1}\left(\mathbb{R}^{N}\right)}+1\right) .
$$

This is not yet what we want; we need inequalities for $\|x\|_{C^{n}\left([0, T] \times B_{r}\right)}$.

To proceed, we first note that as a consequence of standard interpolation inequalities (see, e.g., Theorem A5 in [Hor1]), one can show that for every $\varepsilon>0$, there exists a constant $c(\varepsilon, n, T, r)$ such that

$$
\|x\|_{C^{n}\left([0, T] \times B_{r}\right)} \leq \varepsilon\left\|\frac{\partial x}{\partial t}\right\|_{C^{n}\left([0, T] \times B_{r}\right)}+c(\varepsilon, n, T, r)\|x\|_{C^{n}\left(B_{r}\right)} .
$$

The $\|x\|_{C^{n}\left(B_{r}\right)}$ term on the right hand side of (72) is controlled by the inequalities (71). To control the first term, we use equation (60a) together with the composition inequality (46) to show that

$$
\begin{aligned}
& \left\|\frac{\partial x}{\partial t}\right\|_{C^{n}\left([0, T] \times B_{r}\right)} \\
& =\|H\|_{C^{n}\left([0, T] \times B_{r}\right)} \\
& \leq c\left(\|H\|_{C^{n}\left(\mathbb{R}^{N}\right)}\|x\|_{C^{1}\left([0, T] \times B_{r}\right)}+\|H\|_{C^{1}\left(\mathbb{R}^{N}\right)}\|x\|_{C^{n}\left([0, T] \times B_{r}\right)}\right) .
\end{aligned}
$$

Now $\|x\|_{C^{n}\left([0, T] \times B_{r}\right)}$ appears in (73). However, letting $\kappa$ denote a constant such that $\|H\|_{C^{1}\left(\mathbb{R}^{N}\right)} \leq \kappa$, we find that we can always choose 
$\varepsilon(\kappa, N, T, n, r)$ so that, substituting (72) into (73), we have

$$
\begin{aligned}
& \left\|\frac{\partial x}{\partial t}\right\|_{C^{n}\left([0, T] \times B_{r}\right)} \\
& \quad \leq c\left(\|H\|_{C^{n}\left(\mathbb{R}^{N}\right)}\|x\|_{C^{n}\left([0, T] \times B_{r}\right)}+\|H\|_{C^{1}\left(\mathbb{R}^{N}\right)}\|x\|_{C^{n}\left(B_{r}\right)}+1\right) .
\end{aligned}
$$

Then if we use this inequality (74) for $\left\|\frac{\partial x}{\partial t}\right\|_{C^{n}\left([0, T] \times B_{r}\right)}$ together with the previously obtained inequality (67) for $\|x(t)\|_{C^{1}\left(B_{r}\right)}$, we get from (72) with $\varepsilon=1$ - the result

$$
\|x\|_{C^{n}\left([0, T] \times B_{r}\right)} \leq c\left(T, n, N, r,\|H\|_{C^{1}\left(\mathbb{R}^{N}\right)}\right)\left[\|H\|_{C^{n}\left(\mathbb{R}^{N}\right)}+1\right] .
$$

Thus we obtain a tame estimate for $x(t, \xi)$, and thereby demonstrate that $\Lambda_{[h, Y]}$ is a tame map.

Completion of proof of Theorem 2. Recall that the idea of the proof is to use a Nash-Moser type argument to establish the existence of the function $\theta_{g}: \hat{\mathcal{H}} \rightarrow \mathbb{R}$ (described just below equation (18)). The argument focuses on the study of the function $F$ defined in equation (18), and on the study of the "right inverse" $\alpha=\ell_{[g, f]} \mu$ to the map $D_{2} F$.

In Proposition 3, we have shown that $\alpha$ exists, and we have shown that it may be constructed by setting

(76) $(\alpha \circ \psi)(s, p)$

$$
=\left\{\exp \left[-\int_{0}^{s} \hat{\gamma}(t, p) d t\right]\right\}\left\{\hat{\alpha}(0, p)+\int_{0}^{s} \hat{\mu}(t, p) \exp \left[\int_{0}^{t} \hat{\gamma}(u, p) d u\right] d t\right\}
$$

for

$$
\hat{\alpha}(0, p)=H_{0}+G_{0} G_{1}+\sum_{i=2}^{\infty} G_{0} \cdots G_{i-1} H_{i},
$$

where $H_{i}, G_{i}, \psi, \Psi, \hat{\mu}$, and $\hat{\gamma}$ are as defined in Proposition 3 , and in equation (25).

It follows from Proposition 7 that $\|\alpha(0, p)\|_{\left.C^{n(2} M\right)}$ satisfies an inequality of the form (52), so long as $\mathcal{G}:=\sup _{2} M G$ is smaller than a certain constant $\varepsilon_{n}$. (This $\varepsilon_{n}$ is essentially constructed in the course of proving Lemma 6). Taking $G(p)=\exp \int_{0}^{1} \gamma(t, p) d t$ from equation (33), with $\hat{\gamma}=\gamma \circ \psi$, and with $\gamma=\frac{-\partial g^{\mu \nu}}{\partial x^{0}}\left(x^{0}=-f\left(x^{j}\right), x^{i}\right)\left(x^{0}+f\left(x^{i}\right)\right),{ }_{\mu}\left(x^{0}+f\left(x^{i}\right)\right),{ }_{\nu}$, from equation 
(25), we find that the coordinate-independent expression for the condition $\mathcal{G}<\varepsilon_{n}$ is exactly

$$
Q>\kappa\left(k,\|\Psi\|_{1}\right),
$$

where $Q$ is defined in equation (11), and $k$ (the differentiability of the horizon $\hat{\mathcal{H}})$ replaces $n$. This inequality $(78)$ is, we recall, one of the hypotheses of the Theorem.

We will now show that so long as (78) is satisfied, with the constant $\kappa\left(k,\|\Psi\|_{1}\right)$ to be determined below, then $\theta_{g}$ exists. We do this using Hörmander's version of the Nash-Moser theorem, presented as Theorem 2.2.2 in [Hor1]. Note that to facilitate the verification of the hypotheses of Hörmander's Theorem 2.2.2 - these hypotheses are expressed primarily in the inequalities (2.1.5), (2.1.6), and 2.2.24) in [Hor1] - we will rename some of our functions to match his notation.

Let $\mathcal{O}$ denote the neighborhood of $(\hat{g}, 0) \in \mathcal{M}^{k-1} \times C^{k}(\hat{\mathcal{H}})$ defined just below equation (25), and for every fixed $g$ such that $(g, u) \in \mathcal{O}$, let us set

$$
\Phi_{g}(u):=F(g, u)
$$

with domain $\{u \mid(g, u) \in \mathcal{O}\}$ for $F$ defined as in equation (18). One verifies (with the help of some calculations in [Ham]) that $\Phi_{g}$ satisfies the estimates (2.1.5) in [Hor1] with $\lambda_{0}=0$, with $m_{1}=m_{2}=\cdots=m_{5}=1$, and with $a_{\Phi}=$ $k-1$ (where, as above, $k$ is the differentiability index from the hypotheses of Theorem 2).

Proposition 3 shows that $\Phi_{g}^{\prime}(u)$ - which is the same as $D_{2} F(g, u)$ - has a right inverse. Conforming to Hörmander's notation, we label ${ }^{6}$ this right inverse $\psi_{g}(u):=\ell_{[g, u]}$

As a consequence of Proposition 3 together with Lemma 8, so long as inequality (78) holds, the function $\psi_{g}(u)$ is a composition of tame maps. Hence, as shown by Hamilton (see Lemma 2.1.7 in [Ham]), for certain constants $\mu_{1}, \mu_{2}, \lambda_{1}, \lambda_{2}$, and $a_{\psi}$, the inequality (2.1.6) in [Hor1] is satisfied by $\psi_{g}(u)$.

One way to determine what $\kappa\left(k,\|\Psi\|_{1}\right)$ should be in (78) is to view (2.1.6) in [Hor1] as holding with $\mu_{1}, \mu_{2}, \lambda_{1}$, and $\lambda_{2}$ independent of $k$ and $\|\Psi\|_{1}$, but with $a_{\psi}$ depending upon $k$ and $\|\Psi\|_{1}$ as a consequence of (78). So now let $\alpha_{o}$ be the smallest integer such that $\alpha=\alpha_{0}$ satisfies inequality (2.2.28), in [Hor1], and let $\stackrel{\circ}{a}_{\psi}$ be the smallest integer such that (2.2.24) in [Hor1] is

\footnotetext{
${ }^{6}$ The use of $\psi_{g}(u)$ for this right inverse should not be confused with our use of $\Psi$ to denote the Poincaré map.
} 
satisfied with $a_{\psi}=\stackrel{\circ}{a}_{\psi}$ and $\alpha=\alpha_{0}$. Note that there exists an integer $k_{0}$ such that (2.2.24) in [Hor1] holds with $\alpha=\alpha_{o}$ for all $k \geq k_{0}$ [with $k$ affecting (2.2.24) via the dependence of $a_{\psi}$ on $k$ ]. One finds that there also exists an integer $k_{1}$ satisfying $k_{1} \geq \mu_{1}+\stackrel{\circ}{a}_{\psi}$ and $k_{1} \geq k_{0}$, and there exists a constant $\kappa\left(k_{1},\|\Psi\|_{1}\right)$ such that if $k \geq k_{1}$ and if $Q \geq \kappa\left(k_{1},\|\Psi\|_{1}\right)$, then indeed $\psi_{g}(u)$ satisfies (2.1.6) in [Hor1] with $a_{\psi}=\stackrel{\circ}{a}_{\psi}$. Thus we determine $\kappa\left(k_{1},\|\Psi\|_{1}\right)$ for (78).

Now let us choose some sufficiently small $\sigma>0$ and set $M_{\sigma}=(-\sigma, \sigma) \times \hat{\mathcal{H}}$ in coordinates as discussed early in the proof of this theorem. We consider the set of metrics

$$
\mathcal{O}_{\varepsilon}=\left\{g \mid\|g-\hat{g}\|_{C^{k_{1}\left(M_{\sigma}\right)}} \leq \varepsilon\right\} .
$$

Using what has been shown thus far, we find that there exists $\varepsilon_{0}>0$ such that for all $\varepsilon \leq \varepsilon_{0}$, the estimates (2.1.5) and (2.1.6) in [Hor1] hold, with constants independent of $g$. In particular for any $\delta>0$, there exists $\varepsilon(\delta)>0$ such that if $g \in \mathcal{O}_{\varepsilon(\delta)}$, then

$$
\left\|\Phi_{g}(0)\right\|_{H^{\alpha_{0}+\lambda_{1}}(\hat{\mathcal{H}})} \leq \delta .
$$

We now set $f:=F(g, 0)$. It follows from Theorem 2.2.2 in [Hor1] that there exists some $\delta_{1}>0$ such that if $\|f\|_{H^{\alpha_{0}+\lambda_{1}}}<\delta_{1}$, then there is a solution $u$ to the equation

$$
\begin{aligned}
\Phi_{g}(u) & =\Phi_{g}(0)+f . \\
& =F(g, 0)-F(g, 0) \\
& =0
\end{aligned}
$$

In other words, there exists $\varepsilon_{1}>0$ such that for all $g$ satisfying $\|g-\hat{g}\|_{C^{k}\left(M_{\sigma}\right)}<\varepsilon_{1}$, there is a solution $u$ to the equation

$$
\begin{aligned}
0 & =\Phi_{g}(u) \\
& =F(g, u)
\end{aligned}
$$

Setting $\theta(g)=u$, our desired result follows.

It follows as well from Theorem 2.2.2 in [Hor1] that the map $\theta$ is differentiable, and that the Cauchy horizon $\mathcal{H}$ defined by $\theta_{g}$ in the spacetime $\left(M_{\sigma}, g\right)$ is a $C^{k-k_{2}}$ embedded submanifold for some $k_{2}$. 


\section{On the Sharpness of the Main Result.}

Our main result specifies conditions on a spacetime $(M, \hat{g})$ with a Cauchy horizon which are sufficient to guarantee that all $C^{k}$ perturbations $g$ of $\hat{g}$ admit a Cauchy horizon $\mathcal{H}$, and are sufficient to guarantee that the map $g \rightarrow$ $L_{(k)}(g)=\mathcal{H}$ is differentiable. In $\S 2$, we showed that if one does not impose conditions, then the metric perturbations of a spacetime with a Cauchy horizon may not all admit Cauchy horizons. Here, we discuss an example which shows that there are spacetimes such that if a map $g \rightarrow L_{(k)}(g)$ does exist for some $k \geq 1$, this map $L_{(k)}$ is not a differentiable function of the metric. [We suspect that, in our example, the map does not in fact exist for $k \geq 1$, but we have not yet been able to prove this.] These results show that our main result, Theorem 2 , is sharp.

We consider a one-parameter family of spacetimes $\left({ }^{4} M, g_{q}\right)$ such that

$$
{ }^{4} M=\left(S^{1} \times_{\Psi} T^{2}\right) \times \mathbb{R}^{1}
$$

where $T^{2}$ is the two-torus represented by the standard lattice equivalence relation on $\mathbb{R}^{2}$ (with "[]" denoting the canonical projection from $\mathbb{R}^{2}$ to $T^{2}$ ), where $\Psi$ is the Poincaré map

$$
\Psi([x])=[B x]
$$

with $B=\left(\begin{array}{ll}2 & 1 \\ 1 & 1\end{array}\right)$, and where $\times_{\Psi}$ is the twisted product defined in equation (4); and such that

$$
g_{q}=2 d t d \phi-q t d \phi^{2}+d y^{2}+d z^{2}
$$

where $y$ and $z$ are coordinates on $T^{2}, \phi$ is a coordinate on $S^{1}, t$ is a coordinate on $\mathbb{R}^{1}$, and $q$ is a positive parameter. (See [Ch-I1] for a detailed discussion of such spacetime constructions.)

One readily verifies that for any member of this family of spacetimes, the region $M_{-}=\left\{p \in{ }^{4} M \mid t(p)<0\right\}$ is globally hyperbolic, and the embedded hypersurface $\mathcal{H}=\left\{p \in{ }^{4} M \mid t(p)=0\right\}$ is a Cauchy horizon. Hence, if one fixes a particular value $q_{0} \in(0,+\infty)$ of the parameter, one finds that at least within the family, the existence of a Cauchy horizon is $C^{0}$-stable under $C^{2}$ perturbations of the metric, and we have a well-defined map ${\underset{q}{o}}_{(0)}$ from $g_{q}$ (near $g_{q_{o}}$ in $C^{2}$ topology) to the Cauchy horizon $\mathcal{H}$ in $\left(M, g_{q}\right)$. 
Now say there were a map $\underset{q_{o}}{L_{(}}(k)(k \geq 1)$ taking all $g$ (including $g_{q}$ ) near some fixed $\hat{g}=g_{q_{o}}$ to the corresponding Cauchy horizons. If this map were differentiable, then it would follow that the linear map

$$
\ell_{q_{0}}^{\ell}[g, f]: C^{k}(\mathcal{H}) \rightarrow C^{0}(\mathcal{H})
$$

defined as in Proposition 3 must exist, and its image must lie in $C^{1}(\mathcal{H})$. We shall show that at least for some values of $q_{o}$, the image of $\ell_{q_{0}}[g, f]$ is not contained in $C^{1}(\mathcal{H})$, so it follows that $L_{q_{o}}(1)$, if it exists, is not differentiable.

It is sufficient to show that the function $A: T^{2} \rightarrow \mathbb{R}^{1}$ constructed as in equation (34) - for certain $\hat{g}=g_{q_{o}}$ and for certain choices of smooth $\mu: \mathcal{H} \rightarrow \mathbb{R}^{1}-$ is not $C^{1}$. To show this, it is convenient to focus on points $p \in T^{2}$ given by

$$
p=[\sigma \nu]
$$

where $\nu$ is the eigenvector for the matrix $B=\left(\begin{array}{ll}2 & 1 \\ 1 & 1\end{array}\right)$ which corresponds to the eigenvalue $\lambda_{+}=\frac{1}{2}(3+\sqrt{5})$, and where $\sigma \in[0,+\infty)$. For such points, we obtain from (34)

$$
A([\sigma \nu])=\sum_{i=0}^{\infty} q_{o}^{i} H\left(\left[\lambda_{+}^{i} \sigma \nu\right]\right) .
$$

The function $H$ depends on the choice of the function $\mu$, but from equation (33) we see that for certain $\mu$ we have $H([0])=0$ and $H([p])>0$ for $p \in T^{2} \backslash\{[0]\}$. We assume such a choice, and define the constant

$$
\eta:=\inf _{\tilde{\sigma} \in\left[1, \lambda_{+}\right]} H([\tilde{\sigma} \nu])>0 .
$$

Now let $\sigma \in\left(0, \lambda_{+}^{-1}\right]$. For any such $\sigma$, there exists a positive integer $\nu$ such that $\lambda_{+}^{\nu} \sigma \in\left[1, \lambda_{+}\right]$, and we have from (88)

$$
\begin{aligned}
A([\sigma \nu]) & >q_{o}^{\nu} H\left(\left[\lambda_{+}^{\nu} \sigma \nu\right]\right) \\
& \geq q_{o}^{\nu} \eta .
\end{aligned}
$$

Since $\lambda_{+}^{\nu} \sigma \geq 1$, we have $\nu \ell n \lambda_{+} \geq-\ell n \sigma$, and so

$$
q_{o}^{\nu} \geq \sigma^{\left|\ln q_{0}\right| / \ell n \lambda_{+}} .
$$


(Here, we use the fact $0<q_{0}<1$ ). Combining (90) and (91), we see that

$$
A([\sigma \nu])>\eta \sigma^{\left|\ell n q_{0}\right| / \ell n \lambda_{+}}
$$

thence

$$
\frac{A([\sigma \nu])-A([0])}{\sigma}>\eta \sigma^{\beta}
$$

for $\beta<0$. It follows that $A$ is not $C^{1}$.

Acknowledgements. We thank the Erwin Schroedinger Institute in Vienna for hospitality while portions of this work were being carried out. Partial support for this research has come from NSF grant PHY 92-01196 at Oregon, and from KBN grant 2P30209506.

\section{References.}

[Be] J. Beem, Causality and Cauchy Horizons, Gen. Rel. Grav., 27 (1995), 93-108.

[Ch] P. Chruściel, On Uniqueness in the Large of Solutions of Einstein's Equations ("Strong Cosmic Censorship"), Australian National University Press, Canberra, 1991.

[Ch-I1] P. Chruściel and J. Isenberg, On the Dynamics of Generators of Cauchy Horizons, in Proceedings of the Kananaskis Conference on Chaos in General Relativity, (eds: P. Hobill, A. Burd, and A. Coley), 1994.

[Ch-I2] P. Chruściel and J. Isenberg, Compact Cauchy Horizons and Compact Cauchy Surfaces, in Directions in General Relativity, Vol. 2 , (eds: B.L. $\mathrm{Hu}$ and T.A. Jacobson), Cambridge U. Press, 1993.

[C-I-M] P. Chruściel, J. Isenberg and V. Moncrief, Strong Cosmic Censorship in Polarized Cauchy Spacetimes, Class Qtm. Grav., 7 (1990), 1671-1679.

[Fe] H. Federer, Geometric Measure Theory, Springer, 1967.

[Ham] R. Hamilton, The Inverse Function Theorem of Nash and Moser, Bull. A.M.S., 7 (1982), 65-222.

[Haw1] S. Hawking, Gravitational Radiation from Colliding Black Holes, Phys. Rev. Lett., 26 (1971), 1344-1346.

[Haw2] S. Hawking, Black Holes in General Relativity, Comm. Math. Phys., 26 (1972), 87-101. 
[Haw3] S. Hawking, Chronology Protection Conjecture, Phys. Rev. D., 46 (1992), 603-611.

[Hor1] L. Hörmander, The Boundary Problems of Physical Geodesy, Arch. Rat. Mech. Anal., 62 (1976), 1-52.

[Hor2] L. Hörmander, Implicit Function Theorem, Lecture notes, Stanford Univ., 1977.

[I-M] J. Isenberg and V. Moncrief, Symmetries of Cosmological Cauchy Horizons with Exceptional Orbits, J. Math. Phys., 26 (1985), 1024-1027.

[M] V. Moncrief, The Asymptotic Behavior of Nonlinear Waves near a Cosmological Cauchy Horizon, J. Math. Phys., 30 (1989), 1760-1768.

[M-I] V. Moncrief and J. Isenberg, Symmetries of Cosmological Cauchy Horizons, Comm. Math. Phys.,89 (1983), 387-413.

[P] R. Penrose, Structure of Spacetime, in Battelles Rencontres, (eds: B. DeWitt and J. Wheeler), Benjamin, 1967.

[Th] K. Thorne, Closed Timelike Lines, in Proceedings of the 13-th International Conference on General Relativity and Gravitation, (ed: C. Kozameh), World Press, 1993.

RECEIVED June 25, 1995.

DÉPARTMENT DE MATHÉMATIQUES

FACULTÉ DES SCIENCES

PARC DE GRANDMONT

F 37200 TOURS

FRANCE

CHRUSCIEL@UNIV-TOURS.FR

Department of Mathematics

AND

Institute For TheORETICAL ScIENCE

UNIVERSITY OF OREGON

EUGENE, OR 97403

U.S.A.

JIM@NEWTON.UOREGON.EDU 